\title{
Effective nonlinearities and multi-wavelength second-harmonic generation in modulated quasi-phase-matching gratings
}

\author{
Bang, Ole; Graversen, T. W.; Clausen, Carl A. Balslev
}

Published in:

Nonlinear Optics: Materials, Fundamentals, and Applications

Link to article, DOI:

10.1109/NLO.2000.883609

Publication date:

2000

Document Version

Publisher's PDF, also known as Version of record

Link back to DTU Orbit

Citation (APA):

Bang, O., Graversen, T. W., \& Clausen, C. A. B. (2000). Effective nonlinearities and multi-wavelength secondharmonic generation in modulated quasi-phase-matching gratings. In Nonlinear Optics: Materials, Fundamentals, and Applications (pp. 162-164). https://doi.org/10.1109/NLO.2000.883609

\section{General rights}

Copyright and moral rights for the publications made accessible in the public portal are retained by the authors and/or other copyright owners and it is a condition of accessing publications that users recognise and abide by the legal requirements associated with these rights.

- Users may download and print one copy of any publication from the public portal for the purpose of private study or research.

- You may not further distribute the material or use it for any profit-making activity or commercial gain

- You may freely distribute the URL identifying the publication in the public portal 


\title{
Effective nonlinearities and multi-wavelength second-harmonic generation in modulated quasi-phase-matching gratings
}

\author{
Ole Bang and Torben W. Graversen \\ Department of Mathematical Modelling, Technical University \\ of Denmark, bldg. 305, 2800 Kongens Lyngby, Denmark \\ Tel.: $(+45) 452531$ 08; Fax: (+45) $45931235 ;$ E-mail: ob@imm.dtu.dk \\ Carl Balslev Clausen \\ ATET Labs-Research, 100 Schulz Drive, Red Bank, NJ 07ro1-7033, USA \\ Tel.: 732345 3160; Fax: 732345 3037; E-mail: cbc@research.att.com
}

\begin{abstract}
Quasi-phase-matching gratings induces Kerr effects in quadratic nonlinear materials. We show analytically and confirm numerically how modulating the grating changes the effective quadratic and cubic nonlinearities and allows for multi-wavelength second-harmonic generation. (C) 2000 Optical Society of America

OCIS codes: (050.2770) Gratings; (190.2620) Frequency conversion; (190.4410) Nonlinear optics, parametric processes; (230.4320) Nonlinear optical devices
\end{abstract}

Quasi-phase-matching (QPM) by electric-field poling in ferro-electric materials, such as $\mathrm{LiNbO}_{3}$, is promising due to the possibilities of engineering the photolithographic mask, and thus the QPM grating, without also generating a linear grating. A proper design of the longitudinal grating structure allows for distortion free temporal pulse compression [1], soliton shaping [2], broad-band phase matching [3], multi-wavelength secondharmonic generation (SHG) [4], and an enhanced cascaded phase shift [5]. Transverse patterning can be used for beam-tailoring [6], broad-band SHG [7] and soliton steering [8].

At lowest order the effect of QPM is to eliminate the phase mismatch and average the quadratic (or $\chi^{(2)}$ ) nonlinearity, resulting in an effective $\chi^{(2)}$ nonlinearity experienced by the slowly-varying (on the scale of the coherence length) averaged field, which is reduced by a factor of $\pi / 2$. At the next order QPM induces cubic nonlinear self- and cross-phase modulation terms in the equations for the averaged field [9]. This induced nonlinearity is a result of non phase-matched coupling between the average field and higher order modes [10] and of a fundamentally different nature than the intrinsic material Kerr nonlinearity.

The first order averaged equations with the induced cubic terms provide an elegant tool to study the effect of QPM. It has been shown both analytically and numerically that the induced $\chi^{(3)}$ nonlinearity significantly affects the amplitude and phase modulation of cw waves [11], as well as the properties of solitons [9]. However, in conventional materials with single period QPM, such as $\mathrm{LiNbO}_{3}$, the cubic corrections to the effective quadratic nnnlinearity are typical!' small. Here we shn" a nalytically and verify numerically how the average $\chi^{(2)}$ and $\chi^{(3)}$ nonlinearities can be engineered by modulating the QPM grating, such as, e.g., to make their effects equally strong and important.

We consider a linearly polarized electric field $\vec{E}=\hat{e}\left[E_{1}(z) \exp \left(i k_{1} z-i \omega t\right)+E_{2}(z) \exp \left(i k_{2} z-i 2 \omega t\right)+c . c.\right] / 2$, propagating in a lossless QPM $\chi^{(2)}$ medium under conditions for type I SHG. Then the dynamical equations take the form

$$
i \partial_{z} E_{1}+G(z) \chi_{1} E_{1}^{*} E_{2} \mathrm{e}^{-i \Delta k z}=0, \quad i \partial_{z} E_{2}+G(z) \chi_{2} E_{1}^{2} \mathrm{e}^{i \Delta k z}=0
$$

where $\partial_{z} \equiv d / d z, \chi_{j}=\omega d_{\mathrm{eff}} /\left(n_{j} c\right), E_{1}(z)$ is the slowly varying envelope of the fundamental wave (FW) with frequency $\omega$, refractive index $n_{1}$, and wavevector $k_{1}$, and $E_{2}(z)$ is the second harmonic (SH) with refractive index $n_{2}$ and wavevector $k_{2}(j=1,2)$. The $\chi^{(2)}$ coefficient $d_{\mathrm{eff}}=\left|\chi^{(2)}\right| / 2$ is given in MKS units, and $\Delta k=2 k_{1}-k_{2}$ is the wavevector mismatch. The modulation of the $\chi^{(2)}$ susceptibility is described by the periodic grating function $G(z)$ with unit amplitude and Fourier series $G(z)=\sum_{n} g_{n} \exp [$ inf $f(z)]$, where $g_{n}=0$ for $n$ even and $g_{n}=2 /(i \pi n)$ for $n$ odd, are the coefficients of the unperturbed square grating. We take $f(z)=\kappa_{0} z+\epsilon_{2} \sin \left(\kappa_{2} z\right)$ and consider weakly modulated first order QPM gratings with $L_{2} / \epsilon_{2} \gg L_{0}$, where $L_{2}=2 \pi / \kappa_{2}$ is the modulation period and $L_{0}=\pi / \kappa_{0}$ is the unperturbed domain length. This corresponds to a square grating with a slowly varying local domain length given by $L_{d}(z) \approx \pi / \partial_{z} f=\pi /\left[\kappa_{0}+\epsilon_{2} \kappa_{2} \cos \left(\kappa_{2} z\right)\right]$, as illustrated in Fig. 1(left).

Following the approach of [9] we obtain the equation for the average fields on the shortest scale $L_{0} \ll L_{2}$, 

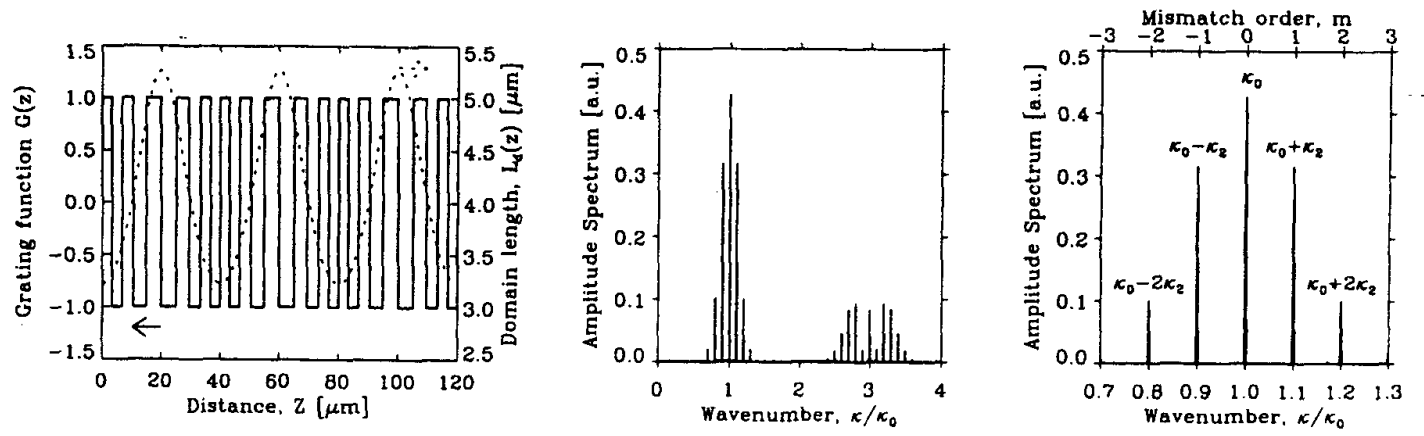

Fig. 1. Left: Modulated grating function $G(z)$ (left,solid) and corresponding local domain length $L_{d}(z)$ (right,dotted) for $L_{2}=10 L_{0}=40 \mu \mathrm{m}$ and $\epsilon_{2}=1.2$. Center: Amplitude spectrum of $G(z)$ for $L_{2}=20 L_{0}=100 \mu \mathrm{m}$ and $\epsilon_{2}=1$.2. Right: Block structure around $\kappa=\kappa_{0}$ and the order $m$ of the effective mismatch $\beta_{m}$.

with induced cubic nonlinear terms and a modulated effective $\chi^{(2)}$ nonlinearity that depends periodically on $z$ (its Fourier coefficients are Bessel functions of the first kind, $J_{n}$ ). Assuming that the modulation length is still much shorter than the crystal length, $L_{2} \ll L$, the spectrum of $G(z)$ has the block structure shown in Fig. 1(center), which allows us to do a second independent averaging on the $L_{2}$-scale. Thus we obtain the final equations for the average fields $w=\left\langle E_{1}\right\rangle$ and $v=\left\langle i E_{2}\right\rangle$, which includes the induced cubic terms:

$$
i \partial_{z} w+\eta_{1 m} w^{*} v \mathrm{e}^{-i \beta_{m} z}+\left(\gamma_{2 m}|w|^{2}-\gamma_{1 m}|v|^{2}\right) w=0, \quad i \partial_{z} v+\eta_{2 m} w^{2} \mathrm{e}^{i \beta_{m} z}-2 \gamma_{2 m}|w|^{2} v=0,
$$

where $\beta_{m}=\Delta k-\kappa_{0}-m \kappa_{2} \ll \kappa_{2}$ is the effective mismatch for matching to the $m$ th peak next to the $\kappa_{0}$ peak, as illustrated in Fig. 1(right). The nonlinearity coefficients are given by

$$
\eta_{j m}=\chi_{j}\left[2 J_{m}\left(\epsilon_{2}\right) / \pi\right], \quad \gamma_{j m}=\chi_{1} \chi_{j}\left[\left(\pi^{2}-8\right) / \kappa_{0}-4 S_{m}\left(\epsilon_{2}\right) / \kappa_{2}\right] / \pi^{2},
$$

where $S_{m}=-S_{-m}=\sum_{n \neq 0} J_{n+m}^{2} / n$. For $m=0$ we obtain $S_{0}=0$. Thus $\gamma_{j 0}=\gamma_{j}$ of the unperturbed grating, as it should be. Using the recurrence and addition formulas for Bessel functions we obtain $S_{1}=-2 J_{0}\left(\epsilon_{2}\right) J_{1}\left(\epsilon_{2}\right) / \epsilon_{2}$. Closed form analytical expressions for $S_{m}$ are progressively more difficult to obtain for higher orders $m \geq 2$. The effective Eqs. (2) for the averaged fields are easily extended to incorporate higher order QPM and diffraction in the transverse $x$ and/or $y$ direction [9].

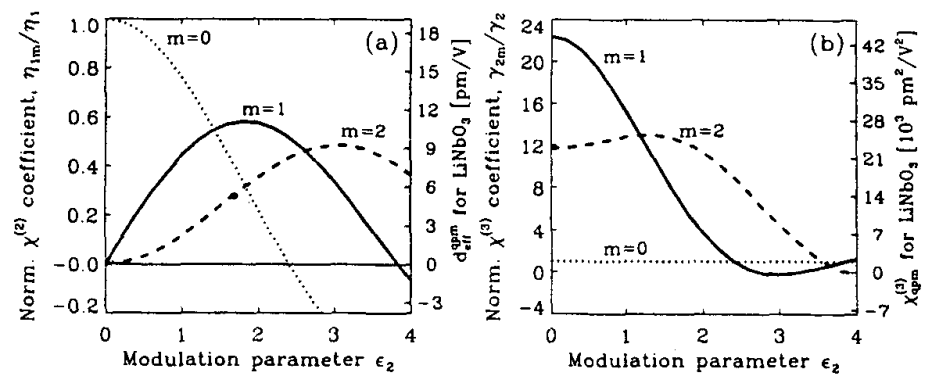

Fig. 2. Normalized average $\chi^{(2)}$ nonlinearity $\eta_{1 m} / \eta_{1}$ (a) and induced cubic nonlinearity $\gamma_{2 m} / \gamma_{2}$ (b) versus modulation parameter $\epsilon_{2}$ for $L_{2}=20 L_{0}=100 \mu \mathrm{m}$. The right ordinate axis shows the strength for bulk $\mathrm{LiNbO}_{3}$.

In Fig. 2 we show the normalized average nonlinearity coefficients $\eta_{1 \mathrm{~m}} / \eta_{1}$ and $\gamma_{2 \mathrm{~m}} / \gamma_{2}$ versus the modulation parameter $\epsilon_{2}$ for different orders of phase matching, and the same QPM grating as in Fig. 1. The right ordinate axis gives the corresponding values for bulk $\mathrm{LiNbO}_{3}$ of the average $\chi^{(2)}$ coefficient $d_{\mathrm{eff}}^{\mathrm{qpm}}$, defined as $\eta_{1 m}=\omega d_{\mathrm{eff}}^{\mathrm{qpm}} /\left(n_{1} c\right)$, and the induced average cubic SPM coefficient $\chi_{\mathrm{qpm}}^{(3)}$, defined as $\chi_{\mathrm{qPm}}^{(3)}=4 n_{1} \lambda_{1} \gamma_{2 m} /(3 \pi)$. We have used a fundamental wavelength of $\lambda_{1}=1.064 \mu \mathrm{m}$, for which $d_{\text {eff }}=30 \mathrm{pm} / \mathrm{V}, n_{1} \simeq n_{2} \simeq 2.2$, and the nonlinear refractive index is $n_{\text {ref }} \simeq 50 \times 10^{-14}$ esu for $\mathrm{LiNbO}_{3}[13]$. From Fig. 2 we see that by matching to the $m=1$ peak we can increase the strength of the induced $\chi^{(3)}$ nonlinearity by a factor of 23 (to $44 \times 10^{3}$ 
$\mathrm{pm}^{2} / \mathrm{V}^{2}$ in $\left.\mathrm{LiNbO}_{3}\right)$ by choosing a sufficiently weak modulation $\left(\epsilon_{2} \ll 1\right)$. In comparison, the material SPM nonlinearity in bulk $\mathrm{LiNbO}_{3}$ is $\chi_{\mathrm{spm}}^{(3)}=3 \cdot 10^{3} \mathrm{pm}^{2} / \mathrm{V}^{2}$, and thus the induced $\chi^{(3)}$ nonlinearity can actually be made dominant by the modulation. However, for $\epsilon_{2} \ll 1$ the effective $\chi^{(2)}$ nonlinearity is reduced (averaged out) to nearly zero for $m \geq 1$. Choosing the right modulation is thus a matter of optimization for the specific design purpose. For example, if the aim is efficient uniform multi-wavelength $S H G$, then $\epsilon_{2} \simeq 1.7$ should be chosen to have a constant value of the effective $\chi^{(2)}$ nonlinearity for all three $m=0,1,2$ peaks.

In conclusion we have shown analytically that modulation of a QPM grating allows to engineer the effective $\chi^{(2)}$ and induced average $\chi^{(3)}$ nonlinearities. We have shown how the induced average $\chi^{(3)}$ nonlinearity can even be made to dominate the intrinsic material one, thereby decreasing the intensity at which the $\chi^{(2)}$ and $\chi^{(3)}$ effects balance.

A whole new range of possibilities arise when $\chi^{(2)}$ and $\chi^{(3)}$ nonlinearities compete on equal footing. Such possibilities include, e.g., engineered bandwidth for parametric wave mixing and frequency generation, cascading phase-shifts, or solitons. In particular, the averaged model can have a self-defocusing cubic nonlinearity, which can dominate the self-focusing one intrinsic to typical $\chi^{(2)}$ materials like $\mathrm{LiNbO}_{3}$, and might therefore support stable dark vortex solitons [14]. This will be the topic of a separate contribution.

In this contribution we will further present numerical verification of the average two-period QPM model (2) and the multi-wavelength SHG spectrum shown in Fig. 1. Such multi-channel wavelength conversion is of considerable technical interest and was recently investigated and confirmed experimentally in $\mathrm{LiNbO}_{3}$ waveguides [15]. The average model provides an elegant tool to analytically calculate the spectrum and the bandwidth for SHG at the different peaks. We will also present these calculations and confirm them numerically.

The authors acknowledge support from the Danish Technical Research Council (Talent Grant no. 9800400).

\section{References}

1. M.A. Arbore, O. Marco, and M.M. Fejer, "Pulse compression during second-harmonic generation in aperiodic quasi-phasematched gratings", Opt. Lett. 22, 865 (1997); M.A. Arbore, A. Galvanauskas, D. Harter, M.H. Chou, and M.M. Fejer, "Engineerable compression of ultrashort pulses by use of second-harmonic generation in chirped-period-poled lithium niobate", Opt. Lett. 22, 1341 (1997).

2. L. Torner, C. Balslev Clausen, and M.M. Fejer, "Adiabatic shaping of quadratic solitons", Opt. Lett. 23, 903 (1998).

3. K. Mizuuchi and K. Yamamoto, "Waveguide second-harmonic generation device with broadened flat quasi-phase-matching response by use of a grating structure with located phase shifts", Opt. Lett. 23, 1880 (1998)

4. S. Zhu, Y. Zhu, Y. Qin, H. Wang, C. Ge, and N. Ming, "Experimental realization of second-harmonic generation in a Fibonacci optical superlattice of $\mathrm{LiTaO}_{3}$ ", Phys. Rev. Lett. 78, 2752 (1997).

5. M. Cha, "Cascaded phase shift and intensity modulation in aperiodic quasi-phase-matched gratings", Opt. Lett. 23, 250 (1998).

6. G. Imeshev, M. Proctor, and M.M. Fejer, "Lateral patterning of nonlinear frequency conversion with transversely varying quasi-phase-matching gratings", Opt. Lett. 23, 673 (1998).

7. P.E. Powers, T.J. Kulp, and S.E. Bisson, "Continuous tuning of a continuous-wave periodically poled lithium niobate optical parametric oscillator by use of a fan out grating design", Opt. Lett. 23, 159 (1998).

8. C. Balslev Clausen and L. Torner, "Self-bouncing of quadratic solitons", Phys. Rev. Lett. 81, 790 (1998); "Spatial switching of quadratic solitons in engineered quasi-phase-matched structures", Opt. Lett. 24, 7 (1999).

9. C. Balslev Clausen, O. Bang, and Y.S. Kivshar, "Spatial solitons and induced Kerr effects in quasi-phase-matched quadratic media", Phys. Rev. Lett. 78, 4749 (1997).

10. O. Bang, Y.S. Kivshar, A, V, Buryak, A. De Rossi, and S. Trillo, "Two-dimensional solitary waves in media with quadratic and cubic nonlinearity", Phys. Rev. E 58, 5057 (1998).

11. A. Kobyakov, F. Lederer, O. Bang, Y.S. Kivshar, "Nonlinear phase shift and all-optical switching in quasi-phase-matched quadratic media", Opt. Lett. 23, 506 (1998)

12. O. Bang, C. Balslev Clausen, P.L. Christiansen, and L. Torner, "Engineering competing nonlinearities", Opt. Lett. 24, 1413 (1999).

13. R. DeSalvo, A.A. Said, D.J. Hagan, E.W. Van Stryland, and M. Sheik-Bahae, "Infrared to ultraviolet measurements of two-photon absorption and $n_{2}$ in wide bandgap solids", IEEE J. Quant Elec. 32, 1324 (1996).

14. T.J. Alexander, A.V. Buryak, and Y.S. Kivshar, "Stabilization of dark and vortex parametric spatial solitons", Opt. Lett. 23, 670 (1998)

15. M.H. Chou, K.R. Parameswaran, M.M. Fejer, and I. Brenner, "Multiple-channel wavelength conversion by use of engineered quasi-phase-matched structures in $\mathrm{LiNbO}_{3}$ waveguides", Opt. Lett. 24, 1157 (1999). 